\title{
LCC from August-November 2018 Lists
}

\section{Compiled by Ann Heinrichs}

The new classifications listed here reflect the most recent information available at the time of publication. Items in this list were selected from the 2018 list numbers 08 (August 20), 09 (September 17), 10 (October 15), and 11 (November 19).

\section{CLASS BL}

[BL619.1582]

BL619.V57

Religions. Mythology. Rationalism

Religion

Worship. Cultus

Rites and ceremonies. Ritual, cult, symbolism

Other, A-Z

Invisibility see BL619.V57

Visibility. Invisibility

History and principles of religion

European. Occidental

Classical religion and mythology

Special deities and characters of classical mythology, A-Z

BL820.G45 Gelos

\author{
Asian. Oriental \\ By religion \\ Hinduism \\ Sacred books. Sources \\ Vedic texts \\ Upaniṣads \\ Individual Upanișads. By title, A-Z
}

BL1124.7.D49-.D499

Dhyānabindūpanișad TABLE BL3

Kalpasūtras (Vedic ritual sūtras)

Śulbasūtras

Individual Sulbasūtras. By title, A-Z

BL1136.8.M35-.M359 Maitrāyaṇiyaśulbasūtra TABLE BL3

Purāṇas

Individual Purānas. By title, A-Z

BL1140.4.S4794-.S47949

Śivadharmottarapurāṇa TABLE BL3

Ann Heinrichs is Metadata/Cataloging Librarian at Catholic Theological Union. 
BL1142.6.S34-.S349

BL1312.9.A73-.A739

BL1357.B43

BL2032.A25

BL2032.A25

[BL2032.I52]

BL2032.M57

BL2211.O24

BL2224.25.C45

BL2236.T74

BL2480.O45
Tantric texts. Śaiva Āgamas. Vaiṣnava saṃhitās

Tantric texts

Individual tantras. By title, A-Z Sạ̣āmnāyatantra TABLE BL3

Jainism

Sacred books. Sources. Āgama (Siddhānta) literature Special divisions and individual texts Paiṇṇas (Prakīṇas) Individual texts. By title, A-Z Ārādhanāpatākā TABLE BL3

Doctrine

Special doctrines, A-Z Bhakti

By region or country India

By ethnic group, etc., A-Z Adivasis CANCEL

Adivasis. Indigenous peoples (General)

Indigenous peoples (General) see BL2032.A25

Miri

Japan

Special topics, A-Z

Ocean

Individual religions

Shinto

Forms of worship. Shinto practice

Ceremonies and rituals Special, A-Z

Chigomai

Korea

Special topics, A-Z

Tree worship

History and principles of religions

African

By ethnic group, etc., A-Z

Okpe 


\section{CLASS BM}

BM729.A88

Judaism

Practical Judaism

Other special topics, A-Z

Attitude (Psychology)

\section{CLASS BP}

BP190.5.P49

Islam. Bahai Faith. Theosophy, etc.

Islam

Topics (not otherwise provided for), A-Z

Physical fitness

\section{CLASS BQ}

BQ649.G83

BQ649.Q23

BQ657

Buddhism

History

By region or country

Asia

East Asia

Special countries

China

Local, A-Z

Guiping Shi

Qiantang River Region

Korea. South Korea

Historiography

Doctrinal and systematic Buddhism

BQ4570.Z66

Special topics (nondoctrinal) and relations to special subjects, A-Z Zombies

Modifications, schools, etc.

Special modifications, sects, etc.

Zen Buddhism

Taehan Pulgyo Chogyejong

Biography

Founders and other important leaders, A-Z

BQ9519.H94-.H949 Hyeam, 1920-2001 TABLE BQ8 


\title{
CLASS BS
}

BS325.A53

BS325.M334

\author{
The Bible \\ General \\ Texts and versions \\ Modern texts and versions \\ Non-European languages \\ African languages, $A-Z$ \\ Amba TABLE BS5 \\ Mankanya TABLE BS5
}

Old Testament

Texts and versions

Modern texts and versions of the Old Testament

English

English versions

Other versions and revisions, $\mathrm{A}-\mathrm{Z}$

BS895.G64-.G642

Goldingay TABLE BS2

\section{CLASS BT}

BT1397

Doctrinal theology

History of specific doctrines and movements. Heresies and schisms

By period

Early to the Reformation, 1517

Luciferians

\section{CLASS BV}

BV4406.86

Practical theology

Pastoral theology

Practical church work. Social work. Work of the layman

Communities and institutions, confraternities, brotherhoods, etc. Individual communities, etc.

Alleluia (Community) TABLE BV8

\section{CLASS DG}

DG457.M66

History of Italy

Medieval and modern Italy, 476-

Ethnography

Individual elements in the population, $\mathrm{A}-\mathrm{Z}$

Moriscos 


\section{CLASS LC}

LC905.M68

Special aspects of education

Moral and religious education

Religion and education. Education under church control

Islamic education

Special topics, A-Z

Motivation

\section{CLASS M}

M2131.C76

Music

Vocal music

Sacred vocal music

Hymnals. Hymn collections

Christian

Published in the United States

1821-

By denomination

Protestant

Other, A-Z

Community of Christ

\section{CLASS PR}

PR428.M367

PR438.J83

English literature

History of English literature

By period

Modern

Elizabethan era (1550-1640)

Other special topics, A-Z

Mary, Blessed Virgin, Saint

17th century

Special topics, A-Z

Judgment Day

\section{CLASS PS}

PS217.A66

American literature

History of American literature

By period

19th century

Special topics, A-Z

Apocalypse 


\title{
CLASS Z
}

Z692.Z56

\author{
Libraries \\ Library science. Information science \\ The collections. The books \\ Special classes of materials \\ Other classes, A-Z \\ Zines \\ Cataloging \\ By subject, A-Z \\ Oceania
}

Z695.1.O33

Machine-readable bibliographic data. Information storage and retrieval systems

By subject or form, $A-Z$

Z699.5.Z56

Zines
Subject bibliography
Manuscripts
By subject, A-Z
Ta'ziyah

Z6611.T23

\author{
Theology and religion \\ Bible \\ Versions, A-Z \\ Italian Bibles \\ Religions (non-Christian) \\ Special, A-Z \\ Ahl-i Hadith
}

Z7771.I8

Z7835.A43

\section{TABLES}

Asia and Eurasia, Africa, Pacific area, and Antarctica: Civil law table (5000 nos.)

KL-KWX4 2695

State and religion

Protection of church property TABLE K11 